\title{
The relationship between isometric mid-thigh pull variables, jump variables and sprint performance in collegiate soccer players
}

\author{
Seita Kuki, Kimitake Sato, Michael H. Stone, Kenichi Okano, Takuya Yoshida, Satoru Tanigawa
}

Objectives: The purpose of this study was to examine the relationship among isometric mid-thigh pull (IMTP) variables, jump variables and sprint times in collegiate soccer players. Additionally, this study was conducted to demonstrate that strength characteristics influence the relationship between jump variables and sprint times.

Design and Methods: Twenty-five collegiate soccer players performed IMTP, jump and sprint assessments. For IMTP, the force output at $100 \mathrm{~ms}(\mathrm{~F} 100 \mathrm{~ms})$ and peak force (PF) were analyzed. Countermovement jump (CMJ) and drop jump (DJ) index were measured. A $30 \mathrm{~m}$ sprint was performed, and the times at $10 \mathrm{~m}, 20 \mathrm{~m}$ and $30 \mathrm{~m}$ were recorded. Pearson's product-moment correlation and a one-way analysis of variance (ANOVA) were used at $\mathrm{p}=0.05$. A cluster analysis was performed to divide all the subjects.

Results: The F100ms significantly correlated with DJ-index $(\mathrm{r}=0.433)$ and sprint times at 20-30m $(\mathrm{r}=-0.444)$. All the subjects were separated into high ( $\mathrm{HG}: \mathrm{N}=9$ ), medium ( $\mathrm{MG}: \mathrm{N}=7$ ) and low ( $\mathrm{LG}: \mathrm{N}=9$ ) groups based on the F100ms, because the coefficient of variation for F100ms was high (34.3\%). There was a strong significant relationship between CMJ and sprint time at $10-20 \mathrm{~m}$ in $\mathrm{HG}(\mathrm{r}=-0.915)$, however there were no significant relationship in MG and LG.

Conclusions: The F100ms can be used as an indicator for identifying athletes who have a statistically significant relationship between CMJ and flying sprint times. The results of the present study suggested that coaches should realize F100ms might provide the foundation to improve the sprint performance.

(Journal of Trainology 2017;6:42-46)

Key words: short sprint performance $\mathbf{a}$ acceleration $\mathbf{m}$ explosive strength $\mathbf{\boldsymbol { \omega }}$ isometric strength

\section{INTRODUCTION}

The total distance covered by soccer players during a match is over $10 \mathrm{~km} .{ }^{1}$ The number of sprints and average distance per sprint are approximately 23 times $^{2}$ and $5-15 \mathrm{~m}^{1}$ respectively, whereas the sprint distance of a match is between $130 \mathrm{~m}$ and $280 \mathrm{~m}$ which is dependent upon playing positions ${ }^{2}$. The sprint speed is an important factor and necessary for superior performance for soccer players, as a study showed that professional soccer players are better sprinters compared to amateur players. $^{3,4}$

A number of studies examined the relationships between sprint performance and strength characteristics including back squat and countermovement jump (CMJ) in soccer players. ${ }^{5,6}$ One study showed 1 repetition maximum (1RM) back squat is strongly correlated with $10 \mathrm{~m}$ sprint time $(\mathrm{r}=-0.94) .{ }^{6}$ Indeed, several studies showed improvement of $20 \mathrm{~m}$ sprint time for soccer players with increased maximum strength. ${ }^{7,8}$ These studies indicate that improving maximum strength plays an important role for sprint performance for soccer players.

Assessing 1RM can be fatiguing and time consuming, whereas an isometric mid-thigh pull (IMTP) may provide a more effective method to assess the force production. The
IMTP described by Haff et al. ${ }^{9}$ is widely used to evaluate the force production in detail. The IMTP is used to measure vertical ground reaction force (GRF) to derive maximum as well as explosive strength such as force at $100 \mathrm{~ms}$ (F100ms) and rate of force development (RFD). ${ }^{10}$ Relationships between IMTP variables and jump performances such as $\mathrm{CMJ},{ }^{11}$ static jump $(\mathrm{SJ})^{12}$ and drop jump (DJ) ${ }^{13}$, and peak power from CMJ were shown to be highly correlated with RFD $(r=0.81)$ and peak force $(r=0.75)$ with from IMTP $^{14}$. For the relationships between IMTP variables and sprint performance, West et al. ${ }^{15}$ showed a moderate correlation $(r=-0.54)$ between the F100ms from IMTP and $10 \mathrm{~m}$ sprint time in rugby players. Although it was clear that the F100ms was moderately correlated with the sprint time from static positon, this study did not examine the split times to evaluate the top speed in flying sprints. Soccer players typically do not sprint from static posture so the flying sprint performance seems more applicable and should be examined. At the same time, the foot contact time during sprinting is approximately $100 \mathrm{~ms}$ to $200 \mathrm{~ms} .{ }^{16-18}$ Therefore, the ability to produce force during short foot contact times may be important factors. The purpose of this study was to examine the relationship among IMTP variables, jump variables, and

Received March 16, 2017; accepted June 29, 2017

From the Graduate School of Comprehensive Human Sciences, University of Tsukuba, Tsukuba City, Ibaraki, Japan (S.K., K.O., T.Y.), Department of Sport, Exercise, Recreation, and Kinesiology, Center of Excellence For Sport science and Coach Education, East Tennessee State University, Johnson City,

TN, USA (K.S., M.H.S.) and Faculty of Health and Sports Science, University of Tsukuba, Tsukuba City, Ibaraki, Japan (S.T.)

Communicated by Takashi Abe, PhD

Correspondence to Mr. Seita Kuki, Graduate School of Comprehensive Human Sciences, University of Tsukuba, Tsukuba City, Ibaraki, Japan. Email: kukiseita0607@gmail.com 
sprint times in collegiate soccer players. Additionally, this study was conducted to analyze the utilization of IMTP variables for training analysis.

\section{Subjects \\ METHODS \\ Twenty-five male college volunteers were recruited from an intercollegiate soccer team with all playing positions, with the exception of goalkeeper, represented (age: $20.20 \pm 0.87$ years, height: $1.72 \pm 0.06 \mathrm{~m}$, weight: $67.12 \pm 6.14 \mathrm{~kg}$ ). All the sub- jects who were free from injury were permitted to participate in this study by the team trainer. The testing protocol was described to each participant in the paper before obtaining informed consent from each subject and in accordance with the guidelines of the University's Institutional Review Board.}

\section{Isometric mid-thigh pull testing}

The IMTP was performed with a custom designed power rack that allows fixation of the bar height. The subjects performed IMTP standing on $60 \times 120 \mathrm{~cm}$ force plate (Ex-Jumper, DKH, Tokyo, Japan), sampled at $1000 \mathrm{~Hz} .{ }^{19}$ Lifting straps and athletic tape were used to remove the influence of grip strength. The body position was determined with knee angle between 125 and 135 degrees, which was assessed using a hand held goniometer. The trunk was in an upright position. ${ }^{9,13}$ Familiarizing the IMTP, each subject performed two practice attempts during the previous day. Two practice attempts at $50 \%$ and $75 \%$ of the subject's perceived maximum effort were performed as warm up before the IMTP testing. ${ }^{20}$ The subjects performed 3 trials with 2 minutes rest between each trial. They were instructed to pull the bar as hard and fast as possible for 6 seconds. They received verbal encouragement during the IMTP.

The vertical GRF during the IMTP was measured. The peak force and the F100ms from the initial point of force increase were determined from the force-time curve. The peak force was defined as peak GRF minus participant's body weight, and the F100ms was defined as the absolute value of GRF from $100 \mathrm{~ms}$ after initial point of the pull minus participant's body weight. ${ }^{15}$ The initiation point of the pull was determined when a GRF exceeding $105 \%$ of the participant's body weight was achieved. ${ }^{21}$ The highest value of both variables in the 3 trials was analyzed. Test-retest reliability for F100ms and PF were shown in Table 1, and it met the standard for reliability which is $\mathrm{ICC}>0.70 .{ }^{22}$

\section{Jump Testing}

After IMTP testing, CMJ and DJ were performed as jump testing. The CMJ was performed on a $60 \times 120 \mathrm{~cm}$ force plate, data sampled at $1000 \mathrm{~Hz}$ (Ex-Jumper, DKH, Tokyo, Japan). The subjects performed 2 trials with hands on hips to minimize the influence of arm swing, and they were instructed to jump as high as possible. The subjects descended from the standing position to a self-selected depth, and jumped with maximal effort. The best of 2 trials was used for analysis. The calculation of the jump height was based on flight time and gravitational acceleration $\left(9.81 \mathrm{~m} \cdot \mathrm{sec}^{-2}\right)$.
The DJ was performed from an upright standing position on a box of $30 \mathrm{~cm}$ height with hands on hips. The subjects stepped off a box and rebounded with the shortest foot contact time and jumped at maximal effort. The foot contact time and the jump height were measured by the switch mat (Multi-Jump Tester, DKH, Tokyo, Japan). The best jump index (DJ-index) of 2 trials was analyzed. The DJ-index was calculated by following equation. ${ }^{23}$ The participants were instructed to minimize alternations in their knee angle at the moment of landing to remove the effect of landing strategy during CMJ and DJ.

$$
\text { DJ-index }(\mathrm{m} / \mathrm{sec})=\text { Jump height }(\mathrm{m}) \cdot \text { Contact time } \mathrm{C}^{-1}(\mathrm{sec}) \text {. }
$$

\section{Sprint Testing}

Forty-eight hours after the first testing day, the $30 \mathrm{~m}$ sprint testing and $10 \mathrm{~m}$ split times were collected. The warm up took about $15 \mathrm{~min}$ including dynamic stretching and 2 attempts of $30 \mathrm{~m}$ sprints as practice. The light gates (TC Timing System, Brower Timing Systems, Utah, USA) were set up at 0 (start), 10,20 and $30 \mathrm{~m}$ (goal). All the participants performed 3 trials with approximately 3 minutes rest between each trial. The subjects started in 2 point standing position from $0.5 \mathrm{~m}$ behind the first gate to prevent early triggering and were instructed to run as fast as possible. The fastest $30 \mathrm{~m}$ sprint time of the 3 trials was analyzed $^{15}$.

\section{Statistical Analysis}

Descriptive statistics were presented as mean and standard deviation (SD). Intraclass correlation coefficients (ICC) and coefficient of variation (CV) were used to assess the reliability of testing. The relationship between IMTP variables, jump variables and sprint times were analyzed using Pearson's product-moment correlation. In order to assess relative strength of the correlation, the scale modified by Hopkins et al. ${ }^{24}$ was used: small $=0.1$ to 0.29 , moderate $=0.30$ to 0.49 , large $=0.50$ to 0.69 , very large $=0.70$ to 0.89 , nearly perfect $=0.90$ to 0.99 and perfect $=1$. A cluster analysis with Ward's method was performed to separate all subjects into three groups based on F100ms. This analysis calculated Euclidian distance based on the F100ms, and separated individuals to high, medium and low group respectively. One-way analysis of variance (ANOVA) was used to determine the difference among three groups. When significant $F$ values were found $(p \leq 0.05)$, post hoc testing were done. The Cohen's d value was calculated based on mean and SD to show practical significance. The statistical analysis for the Pearson's correlation, the cluster analysis, and the ANOVA were performed using SPSS v22 software (IBM, New York, USA). The criterion for statistical significance was considered as $\mathrm{p} \leq 0.05$.

\section{RESULTS}

The descriptive data included mean, SD, CV and ICC for all variables are shown in Table 1 . The F100ms was moderately correlated with DJ-index $(\mathrm{r}=0.433, \mathrm{p}<0.05)$. The F100ms was also correlated with sprint times at $10 \mathrm{~m}(\mathrm{r}=-0.521$, $\mathrm{p}<0.01), 30 \mathrm{~m}(\mathrm{r}=-0.417, \mathrm{p}<0.05)$ and $20-30 \mathrm{~m}(\mathrm{r}=-0.444$, $\mathrm{p}<0.05)$ respectively. 
Table 1 Performance characteristics and reliability.*

\begin{tabular}{llccc}
\hline Test & Variable & Mean $\pm \mathrm{SD}$ & $\mathrm{CV}(\%)$ & ICC \\
\hline Isometric & F100ms (N) & $736.68 \pm 269.17$ & 36.5 & 0.71 \\
mid-thigh pull & Peak Force (N) & $2067.16 \pm 325.07$ & 15.7 & 0.83 \\
\hline \multirow{3}{*}{ Jump } & CMJ (cm) & $39.97 \pm 5.00$ & 12.5 & 0.93 \\
& DJ-index (m/sec) & $1.77 \pm 0.37$ & 20.7 & 0.87 \\
& DJ-height (cm) & $31.28 \pm 5.22$ & 16.7 & 0.89 \\
& DJ-contact time (sec) & $0.19 \pm 0.07$ & 12.5 & 0.84 \\
\hline \multirow{3}{*}{ Sprint } & Sprint 0-10 m (sec) & $1.66 \pm 0.06$ & 3.8 & 0.75 \\
& Sprint 0-20 m (sec) & $2.92 \pm 0.11$ & 3.7 & 0.87 \\
& Sprint 0-30 m (sec) & $4.11 \pm 0.16$ & 4.0 & 0.94 \\
& Sprint 10-20 m (sec) & $1.26 \pm 0.06$ & 4.7 & 0.75 \\
& Sprint 20-30 m (sec) & $1.19 \pm 0.06$ & 5.2 & 0.81 \\
\hline
\end{tabular}

* $\mathrm{CV}=$ coefficient of variation; ICC = intraclass correlation coefficient; $\mathrm{F} 100 \mathrm{~ms}=$ the force output at $100 \mathrm{~ms}$ from initial point of the pull;

$\mathrm{CMJ}=$ countermovement jump; DJ = drop jump.

Although there were statistically significant relationships between the F100ms for IMTP and sprint times, the CV for the F100ms were much higher than criteria for acceptable reliability $^{22}$ as shown in Table 1 . These data suggest that the ability to produce force at $100 \mathrm{~ms}$ was highly dependent on the individual athlete. Therefore, a cluster analysis was conducted to separate those subjects to three $100 \mathrm{~ms}$ force groups; high group (HG: $\mathrm{N}=9$ ), medium group (MG: $\mathrm{N}=7$ ), and low group (LG:
$\mathrm{N}=9$ ). As the result, $\mathrm{CVs}$ for $\mathrm{F} 100 \mathrm{~ms}$ were within criteria for acceptable reliability as follows: $15 \%$ in the LG, $8 \%$ in $\mathrm{MG}$, $13 \%$ in HG respectively. The descriptive data for each group and the comparison among three groups in all variables were shown in Table 2. There were statistical differences observed in $\mathrm{F} 100 \mathrm{~ms}(\mathrm{~F}[2,22]=89.839, \mathrm{p}<0.01), \mathrm{PF}(\mathrm{F}[2,22]=4.552$, $\mathrm{p}<0.05)$, DJ-index $(\mathrm{F}[2,22]=4.233, \mathrm{p}<0.05)$ and sprint time at $0-10 \mathrm{~m}(\mathrm{~F}[2,22]=3.547, \mathrm{p}<0.05)$. For the $\mathrm{F} 100 \mathrm{~ms}$, HG was

Table 2 Performance characteristics in each group.*

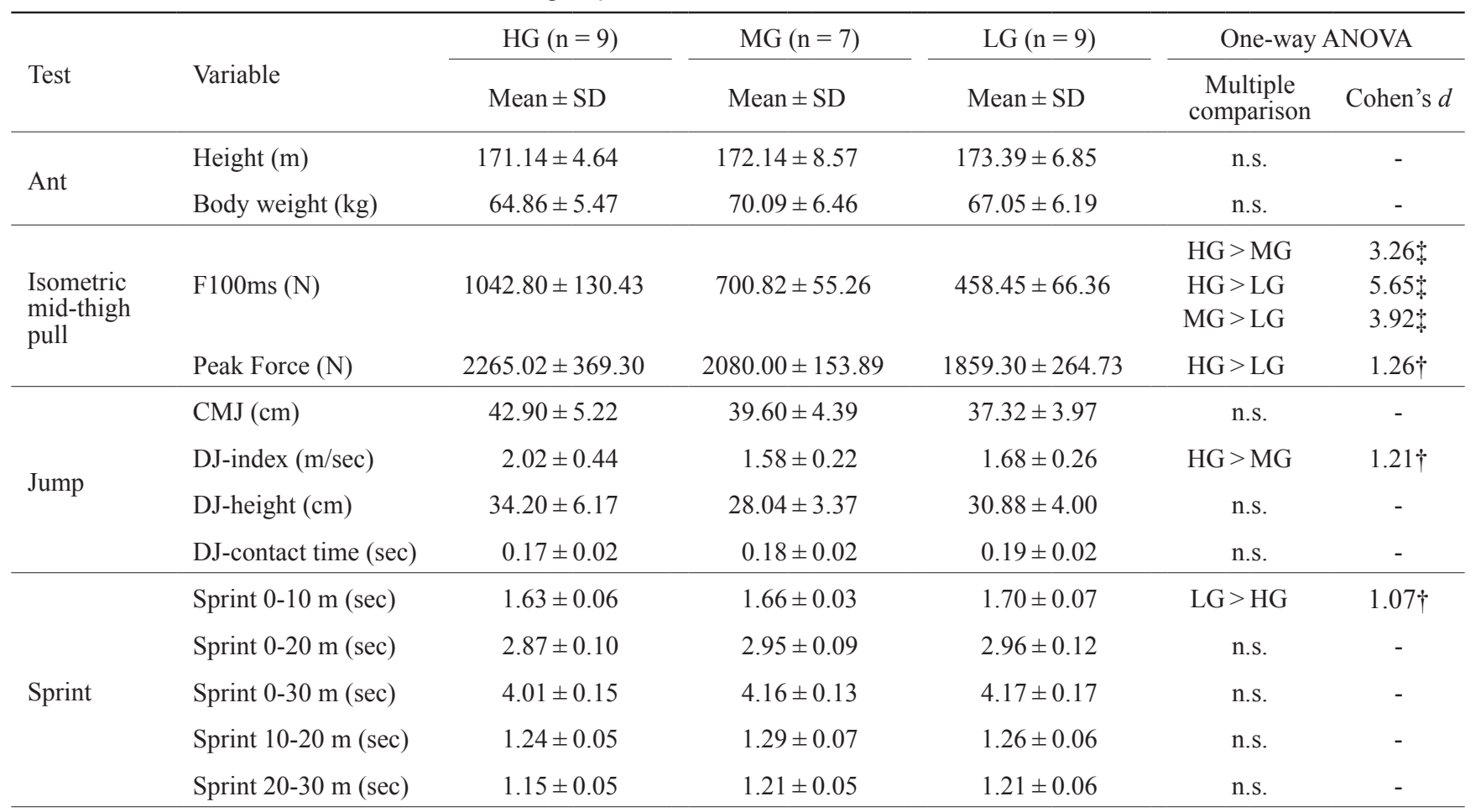

* $\mathrm{HG}=$ high group in the force output at $100 \mathrm{~ms}$ of IMTP; MG = medium group in the force output at $100 \mathrm{~ms}$ of IMTP; LG $=$ low group in the force output at $100 \mathrm{~ms}$ of IMTP; F100ms = the force output at $100 \mathrm{~ms}$ from initiation point of the pull; CMJ = countermovement jump; DJ = drop jump. $\dagger=$ significantly difference $(\mathrm{p}<0.05)$. $\$=$ significantly difference $(\mathrm{p}<0.01)$. 
statistically higher than MG $(\mathrm{d}=3.26, \mathrm{p}<0.01)$ and LG $(\mathrm{d}=$ $5.65, \mathrm{p}<0.01)$, MG was also statistically higher than LG $(d=3.92, p<0.01)$. In the peak force, HG was significantly higher than LG $(\mathrm{d}=1.26, \mathrm{p}<0.05)$. HG was also statistically higher than MG in DJ-index $(\mathrm{d}=1.21, \mathrm{p}<0.05)$ and faster than LG in sprint time at $0-10 \mathrm{~m}(\mathrm{~d}=1.07, \mathrm{p}<0.05)$. The relationship among the IMTP variables, jump variables, and sprint times for each group were analyzed. There were no statistically significant relationships between the F100ms and sprint times in each group, but there were strong statistically significant relationships between CMJ and sprint times: $10-20 \mathrm{~m}$ $(\mathrm{r}=-0.915, \mathrm{p}<0.01)$ and $20-30 \mathrm{~m}(\mathrm{r}=-0.764, \mathrm{p}<0.05)$ in $\mathrm{HG}$ and $20-30 \mathrm{~m}(\mathrm{r}=-0.775, \mathrm{p}<0.05)$ in $\mathrm{MG}$.

\section{DISCUSSION}

The purpose of this study was to examine the relationship among IMTP variables, jump variables and sprint times in soccer players, and to propose how to utilize IMTP variables for analyzing/modifying training to enhance sprint performance. Some of the results from the current study corresponded with previous study that indicated a significant relationship between F100ms and sprint time for $10 \mathrm{~m} .{ }^{15}$ The ability to produce high force momentarily is quite important, because it allows for maximal force production with relatively short foot contact times (i.e. $100 \mathrm{~ms}$ ), ultimately resulting high acceleration. The primary finding of the current study was that F100ms was significantly correlated with sprint times. Based on the current study, the F100ms could be an indicator for identifying athletes who have a significant relationship between CMJ and flying sprint performance Correlation analysis indicated that relationships between $\mathrm{CMJ}$ and flying sprint times in $\mathrm{HG}$ were much higher than MG and LG. Particularly for HG, the correlation between $\mathrm{CMJ}$ and flying sprint time at $10-20 \mathrm{~m}$ was very high $(r=-0.915)$, indicating that the F100ms is the most useful variable identified to aid in ascertaining the relationship of vertical jump performance and flying sprint performance. Thus, the F100ms would be considered as prerequisite for identifying the strong relationship between CMJ and flying sprint times. Although some previous studies also demonstrated the significant relationship between $\mathrm{CMJ}$ and sprint time in soccer players, ${ }^{5,6}$ the influence of individual strength on this relationship has yet to reported. The current study has provided additional evidence that sprint ability is dependent upon a variety of physical qualities.

Jump training is usually incorporated to enhance sprint performance. However, poor strength may increase the chance of poor performance development or injury from jump training, ${ }^{25}$ so that strength could be considered as a fundamental for jump training. Strength development for the prevention of injuries from jump training has been recommended ${ }^{25,26}$. Moreover, athletes with high relative strength could effectively improve jump performance through power training, such as jump squats, as compared with athletes with low relative strength. ${ }^{27}$ Establishment of the solid foundation of strength prior to power training would be recommended. ${ }^{27}$ The current study showed that the relationships between $\mathrm{CMJ}$ and sprint times were weak in LG. Therefore, there is a possibility that the
F100ms plays a role to potentially identify the minimum requirement to enhance sprint performance effectively by vertical jump training. Strength training, including back squats with $75 \%-90 \% 1 \mathrm{RM}$ for 10 weeks, significantly improves relative strength for relatively weak participants. ${ }^{28}$ Therefore the athletes with low F100ms should focus on strength training prior to starting jump training even during relatively short training periods.

There are some limitations for this study. Firstly, the CV of the F100ms was much higher than other testing variables (Table 1). The previous studies that examined the F100ms also demonstrated the high $\mathrm{CV}$, which it was ranged $25-43 \%{ }^{10,19} \mathrm{~A}$ lack of familiarization to perform IMTP may be the reason of this high value of $\mathrm{CV}$, although a previous study demonstrated that the familiarization of the IMTP was enough at two practices with submaximal effort as warm-up..$^{29}$ Secondly, the jump heights for CMJ and DJ were calculated by flight times, whereas calculations based on GRF may provide more accurate calculations.

\section{CONCLUSION}

The primary finding of this study was the significant relationship between F100ms and flying sprint time at 20-30m. Furthermore, all the subjects were separated to three groups by F100ms, and the relationship between CMJ and sprint time was examined. In $\mathrm{HG}$, there was strong significant relationship between CMJ and flying sprint time at $10-20 \mathrm{~m}$, although there were no significant relationships in MG and LG. Therefore the F100ms can be used as an indicator for identifying athletes who have a statistically significant relationship between CMJ and flying sprint times. These results represent options for coaches to evaluate athletes and create individualized training programs using IMTP testing.

\section{COMPLIANCE}

No funding was received for this study from any organization.

\section{REFERENCE}

1. Bangsbo J, Mohr M, Krustrup P. Physical and metabolic demands of training and match-play in the elite football player. J Sports Sci 2006;24: 665674.

2. Salvo VD, Baron R, Haro CG et al. Sprinting analysis of elite soccer players during European champions league and UEFA cup matches. $J$ Sports Sci 2010;28:1489-14946.

3. Barnes C, Archer DT, Bush M et al. The evolution of physical and technical performance parameters in the English premier league. Int J Sports Med 2014;35:1095-1100.

4. Cometti G, Maffiuletti N, Pousson M et al. Isokinetic strength and anaerobic power of elite, subelite and amateur French soccer players. Int J Sports Med 2011;22:45-51.

5. Comfort P, Stewart A, Bloom L et al. Relationships between strength, sprint, and jump performance in well-trained youth soccer players. $J$ Strength Cond Res 2014;28:173-177.

6. Wisloff U, Castagna C, Helgerud J et al. Strong correlation of maximal squat strength with sprint performance and vertical jump height in elite soccer players. J Sports Med 2004;38:285-288.

7. Sander A, Keiner M, Wirth K et al. Influence of a 2-year strength training programme on power performance in elite youth soccer players. Eur $J$ Sport Sci 2012;13:445-451 
8. Styles WJ, Matthews MJ, Comfort P. Effects of strength training on squat and sprint performance in soccer players. J Strength Cond Res 2016;30: 1534-1539.

9. Haff GG, Stone MH, O’Bryant, HS et al. Force-time dependent characteristics of dynamic and isometric muscle actions. J Strength Cond Res 1997; 11:269-272.

10. Wang R, Hoffman JR, Tanigawa $S$ et al. Isometric mid-thigh pull correlates with strength, sprint and agility performance in collegiate rugby union players. J Strength Cond Res 2016;30:3051-3056.

11. Kawamori N, Rossi SJ, Justice BD et al. Peak force and rate of force development during isometric and dynamic mid-thigh clean pulls performance at various intensities. J Strength Cond Res 2006;20:483-491.

12. Haff GG, Carlock JM, Hartman MJ et al. Force-time curve characteristics of dynamic and isometric muscle actions of elite women Olympic weightlifters. J Strength Cond Res 2005;19:741-748.

13. Beattie K, Carson BP, Lyons M et al. The relationship between maximalstrength and reactive-strength. Int J Sports Physiol Perform 2017;12:548553.

14. Nuzzo JL, McBride JM, Cormie, $P$ et al. The relationship between countermovement jump performance and multijoint isometric and dynamic tests of strength. J Strength Cond Res 2008;22:699-707.

15. West DJ, Owen NJ, Jones MR et al. Relationships between force-time characteristics of the isometric mid-thigh pull and dynamic performance in professional rugby league players. J Strength Cond Res 2011;25:30703075 .

16. Mero A, Komi PV, Gregor RJ. Biomechanics of sprint running. Sports Med 1992;13:376-392.

17. Morin JB, Slawinski J, Dorel S et al. Accelrration capability in elite sprinters and ground impulse: push more, brake less? J Biomech 2015;48:31493154.

18. Spinks CD, Murphy AJ, Spinks WL et al. The effects of resisted sprint training on acceleration performance and kinematics in soccer, rugby union, and Australian football players. J Strength Cond Res 2007;21:77-85.
19. Leary BK, Statler J, Hopkins B et al. The relationship between isometric force-time curve characteristics and club head speed in recreational golfers. J Strength Cond Res 2012;26:2685-2697.

20. Beckham G, Mizuguchi S, Carter C et al. Relationships of isometric midthigh pull variables to weightlifting performance. J Sports Med Phys Fitness 2013;53:573-581.

21. Dos'Santos T, Jones PA, Comfort P et al. Effect of different onset thresholds on isometric mid-thigh pull force-time variables. J Strength Cond Res, in press.

22. Haff GG, Ruben RP, Lider J et al. A compareson of methods for determining the rate of force development during isometric mid-thigh clean pulls. $J$ Strength Cond Res 2015;29:386-395.

23. Miura K, Yamamoto M, Tamaki H et al. Determinants of the abilities to jump higher and shorten the contact time in a running 1-legged vertical jump in basketball. J Strength Cond Res 2010;24:201-206.

24. Hopkins WB, Marshall SW, Batterham AM et al. Progressive statistics for studies in sports medicine and exercise science. Med Sci Sports Exerc 2009;41:3-12.

25. Suchomel TJ, Nimphius S, Stone MH. The importance of muscular strength in athletic performance. Sports Med 2016;46:1419-1449.

26. Jacobs C, Mattacola C. Sex differences in eccentric hip-abductor strength and knee-joint kinematics when landing from a jump. J Sports Rehab 2005;14:346-355.

27. Cormie P, McGuigan MR, Newton RU. Influence of strength on magnitude and mechanisms of adaptation to power training. Med Sci Sports Exerc 2010;42:1566-1581.

28. Cormie P, McGuigan MR, Newton RU. Adaptations in athletic performance after ballistic power versus strength training. Med Sci Sports Exerc 2010;42:1582-1598.

29. Beckham G, Sato K, Stone MH et al. The effect of various body positions on performance of the isometric mid-thigh pull. The faculty of the department of exercises and sport sciences East Tennessee State University, Dissertation, 2015. 